\title{
Seborrheic Dermatitis
}

National Cancer Institute

\section{Source}

National Cancer Institute. Seborrheic Dermatitis. NCI Thesaurus. Code C111888.

A chronic, inflammatory skin disorder that affects the scalp, central face and skin folds; it

is characterized by scaling and itching. 